\title{
A multi-sensor scenario for coastal surveillance
}

\author{
A.C. van den Broek, S.P. van den Broek, J.C. van den Heuvel, P.B.W. Schwering, A.W.P. van \\ Heijningen, TNO Defence, Security and Safety, P.O. Box 96864, 2509 JG, The Hague, The \\ Netherlands, bert.vandenbroek@tno.nl
}

\begin{abstract}
Maritime borders and coastal zones are susceptible to threats such as drug trafficking, piracy, undermining economical activities. At TNO Defence, Security and Safety various studies aim at improving situational awareness in a coastal zone. In this study we focus on multi-sensor surveillance of the coastal environment. We present a study on improving classification results for small sea surface targets using an advanced sensor suite and a scenario in which a small boat is approaching the coast.

A next generation sensor suite mounted on a tower has been defined consisting of a maritime surveillance and tracking radar system, capable of producing range profiles and ISAR imagery of ships, an advanced infrared camera and a laser range profiler. For this suite we have developed a multi-sensor classification procedure, which is used to evaluate the capabilities for recognizing and identifying non-cooperative ships in coastal waters.

We have found that the different sensors give complementary information. Each sensor has its own specific distance range in which it contributes most. A multi-sensor approach reduces the number of misclassifications and reliable classification results are obtained earlier compared to a single sensor approach.
\end{abstract}

Keywords: Target recognition, littoral, surveillance, sensor fusion

\section{INTRODUCTION}

Effective surveillance of maritime borders and coastal zones is a necessity in case of illegal activities. For this purpose a variety of sensor information can be used such as radars, cameras (visual and infrared), and lasers, with optional support from satellites imagery. In addition, AIS (Automatic Identification System) can be used to discriminate cooperative targets from non-cooperative targets.

The Single Picture Integration for Territorial water Surveillance (SPITS, Theil et al., 2006) initiative at TNO Defence, Security and Safety aims at improving situational awareness in a coastal environment by detecting anomalous behaviour of surface and air targets. In this paper we focus on improving classification results for small sea surface targets. For this purpose a scenario for multi-sensor surveillance of the coastal environment has been studied. A next generation sensor suite consisting of a maritime surveillance and tracking radar system, capable of producing range profiles and ISAR (Inverse Synthetic Aperture Radar) imagery of ships, an infrared camera and a laser range profiler is defined. On the basis of simulated data for this sensor suite improved capabilities for recognizing and identifying non-cooperative ships in coastal waters are demonstrated, in such a way that the quality of the maritime surface picture in the littoral environment can be enhanced.

The scenario is as follows: the radar system detects and tracks ships, while simultaneously information from the sensor suite is extracted. This information is first used to select suspect ships. Next, information for the selected ships is compared with information about ships in a database. On the basis of this comparison and the use of a priori information, probabilities are obtained for suspect ships.

By observing such non-cooperative ships, for example during an approach to the coast, the information from the multisensor suite can be combined in order to produce improved recognition performance.

Unmanned/Unattended Sensors and Sensor Networks IV, edited by Edward M. Carapezza,

Proc. of SPIE Vol. 6736, 67360P, (2007) · 0277-786X/07/\$18 · doi: 10.1117/12.736646

Proc. of SPIE Vol. 6736 67360P-1 
The paper is organized as follows. In Section 2 we describe the scenario and sensor suite. In Section 3 we describe the processing of the sensor data, which are obtained through modelling. In Section 4 we present results from a workflow for multi-sensor classification. In Section 5 we give discussions and conclusions.

\section{SCENARIO AND MULTI-SENSOR SUITE}

The following scenario is defined, representing for example smugglers landing on a beach. Sensors are located on an observation tower with a height of 40 meter and with a distance of 100 meter from the coastline. A ship is approaching the coast with a speed of 10 knots and with an angle of 75 degrees to the coast (see Figure 2.1). The situation is at night and hence an infrared (IR) camera is indispensable compared to optical cameras. We consider the atmospheric conditions as optimal with an attenuation of maximally $0.1 / \mathrm{km}$ in the infrared.

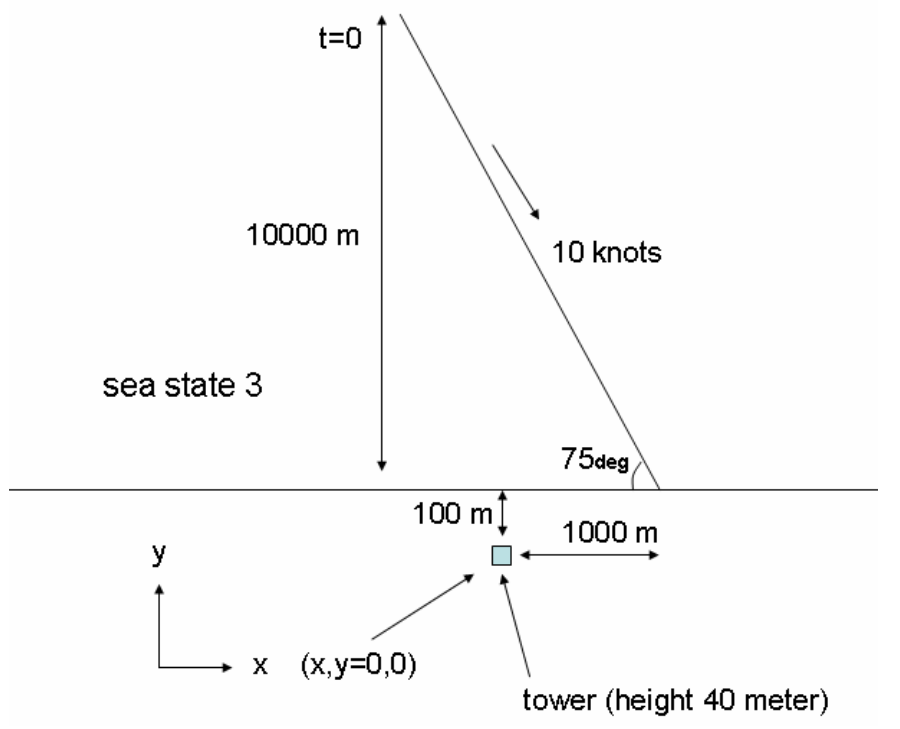

Figure 2.1. Layout of scenario

The scenario is for shallow waters like in the North Sea. A sea state category three is chosen which implies wave heights in the order of one meter, wavelengths in the order of 70 meter and periods of 7-10 seconds in such waters. These waves force floating platforms like ships to have periodically varying pitch, yaw and roll angles. We assume here no yaw. Pitch and roll angles are in the order of a few (2-4) degrees. The pose of the platform is determined by an azimuth and elevation angle. Most of the variation due to the waves is found for the elevation angle (see Figure 2.2). This elevation angle is defined here as the angle between the line of sight and the mast of the ship. This variation in angle is important to obtain sufficient resolution in cross-range direction in the ISAR imagery.

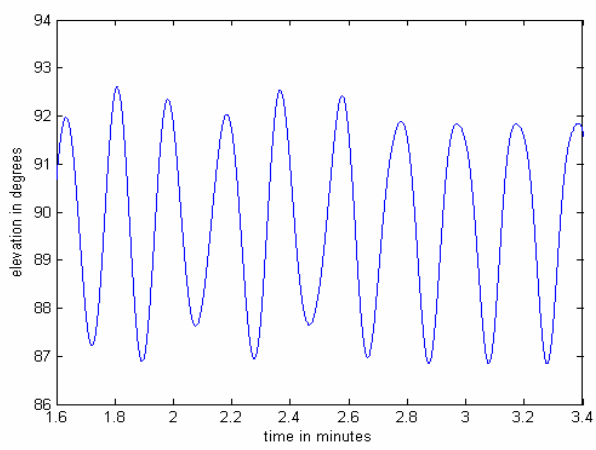

Figure 2.2. Variation in elevation angle for a time interval of 2 minutes 
For the multi-sensor suite we have defined sensors that we expect to become affordable and on the commercial market within a few years. The sensor suite comprises a maritime radar, an IR camera and a laser range profiler. These are described below.

The maritime radar can be a single radar with a surveillance and tracking mode or can consist of two separate radars, one for surveillance to detect targets and one for tracking and producing ISAR imagery. Since the surveillance radar can be a conventional radar we discuss here the tracking radar. This radar can use monopulse techniques (Levanon, 1988) to increase the tracking accuracy (in the order of 0.1 degree). The radar uses X-band (10 GHz, VV polarisation) with a bandwidth of $500 \mathrm{MHz}$ (30 cm range resolution), with a peak power of $1000 \mathrm{~W}$ for emitted pulses, and with a pulse repetition frequency (PRF) of $1 \mathrm{kHz}$, which is sufficient for ISAR imaging.

For the IR camera a state-of-the art cooled system was chosen, avoiding the $\mathrm{CO}_{2}$ absorption dip. It operates at a midinfrared wavelength of 3.2-4.2 micron, with an instantaneous field of view (IFOV) of $0.05 \mathrm{mrad}$ and a sensitivity of 10 $\mathrm{mK}$. This waveband is chosen to have good visibility also in warm atmospheric regions. Future extension, making use of longwave infrared uncooled sensors would be feasible for specific (colder and close range) scenarios.

The laser range profiles is based on currently available technology. The proposed laser range profiler operates at 1.57 micron, producing 10 pulses per second with a pulse energy of $30 \mathrm{~mJ}$ and a pulse length of $5 \mathrm{~ns}$. The range resolution of $30 \mathrm{~cm}$ is based on a $200 \mathrm{MHz}$ bandwidth detector currently available with InGaAs APD detectors and minor deconvolution of the combined laser pulse length and detector response.

\section{SENSOR DATA AND PROCESSING}

\subsection{Modelling of sensor data.}

The data for the sensor are obtained through modelling, where the targets are represented by so-called facet models. We have used models for three boats: a Boghammer, a Cabin boat and a Rhib (Rigid hull inflatable boat). The Boghammer is originally a high speed patrol boat designed by a Swedish company but is nowadays considered as typical for hostile boats that might be used by irregular groups (swarming attacks) in coastal waters. The Cabin boat is a typical small civilian ship which can be found in littoral waters. The Rhib is a small fast boat which can transport several people and is typical for bridging the coast and larger ships, which have to stay off-shore. They are often stored on board of military vessels, such as frigates and are also used for life saving. In Figure 3.1 we show pictures of the boats. Table 3.1 summarizes the main dimensions of the boats.
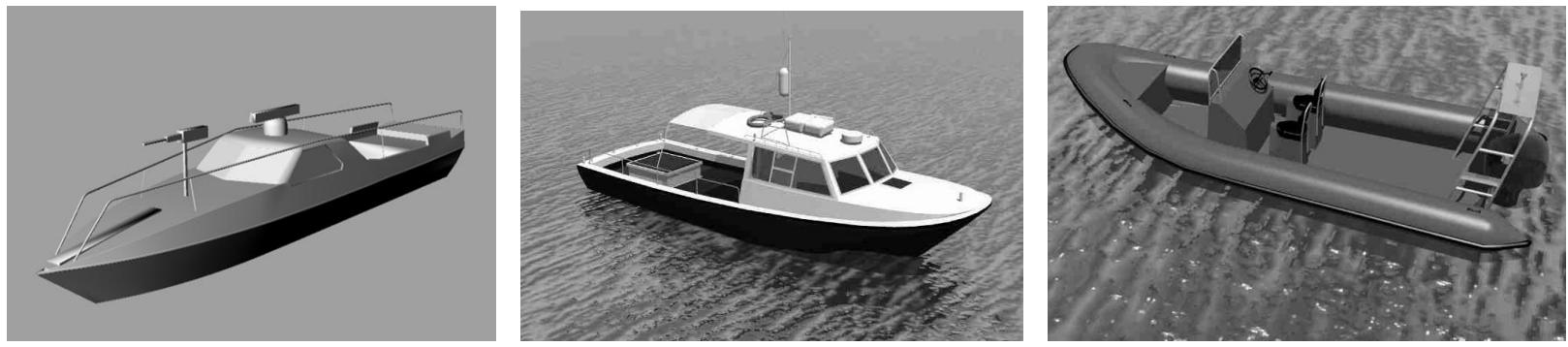

Figure 3.1. Pictures of the Boghammer (left), Cabin boat (middle) and Rhib (bottom)

Table 3.1. Dimensions of the targets.

\begin{tabular}{|l|l|l|l|}
\hline & Length $(\mathrm{m})$ & Width $(\mathrm{m})$ & Height $(\mathrm{m})$ \\
\hline Boghammer & 12.8 & 2.7 & 3.0 \\
\hline Cabin boat & 10.5 & 3.3 & 3.7 \\
\hline Rhib & 6.8 & 2.6 & 1.0 \\
\hline
\end{tabular}


To calculate radar data we have used the radar cross section (RCS) prediction code RAPPORT (Radar signature Analysis and Prediction by Physical Optics and Ray Tracing), which is developed by TNO (van Ewijk, 1992, Brand, 1995). It is a high-frequency technique, implying that it is suitable for complex objects that are much larger than the wavelength of the radar radiation. It is used to predict the RCS of targets, such as ships, vehicles and aircrafts, to generate inverse synthetic aperture radar (ISAR) images of such targets, and to evaluate the effect of RCS reduction measures. The implemented algorithm is based upon a combination of Physical Optics (PO) and Geometrical Optics (GO), as proposed in Knott (1981). Objects have to be described as a collection of flat polygonal plates (facets), because of the adopted method to solve the PO integral (Gordon, 1975). The code takes into account the polarization of the radiation and multiple reflections. In this case however no reflections between the ships and the water surface are calculated. Also shielding of the targets by waves at very shallow grazing angles is not taken into account. The latter is also true for the modelling of the laser and infrared data.

In the infrared modelling the EOSM code (Neele, 2005) is used to calculate the IR signature of the boats, while the EOSTAR code (Kunz et al., 2004) is used to calculate the influence of the atmosphere and the environment on the infrared data which are observed by the camera. Background intensities and clutter is calculated with the MIBS (Schwering, 2006) model. These three models are TNO designs. The EOSM model uses a facet model of the boat and calculates the heat exchange to predict the infrared signatures. EOSTAR calculates the atmospheric corrections for transmission, turbulence, background intensity and refraction to generate the target contrast. MIBS generates a background map of the sea surfaces including intensity variations due to the surfaces waviness and clutter due to whitecaps. MIBS is used in scene generation for near-horizon targets. New background features such as wake estimation and other interactions of the target with the sea surface are not yet implemented. Although the combination of the three models yields realistic looking images (see for example Figure 3.2 right), they appeared to be too ideal for applying an automatic detection algorithm (van den Broek et al., 2000). Instead, target information is used more directly, and errors for derived features are added to make results realistic.

For the laser range profiler, simulated range profiles are obtained from EOSTAR and the facet model. Diffuse Lambertian reflection was assumed for all surfaces. The effect of diffuse reflection and the receiver noise was calculated in MATLAB (http://www.mathworks.com/). The reflection from the sea surface was assumed negligible, which has been confirmed by several experiments in the past near the coast.

From the scenario observation parameters (line-of-sight, distances, angles for elevation and azimuth) are extracted. Using abovementioned models simulated sensor data are obtained for the sensors in the sensor suite observing the three targets. Contributions from the sea surface are ignored. Examples of data for the Cabin boat are shown in Figure 3.2.
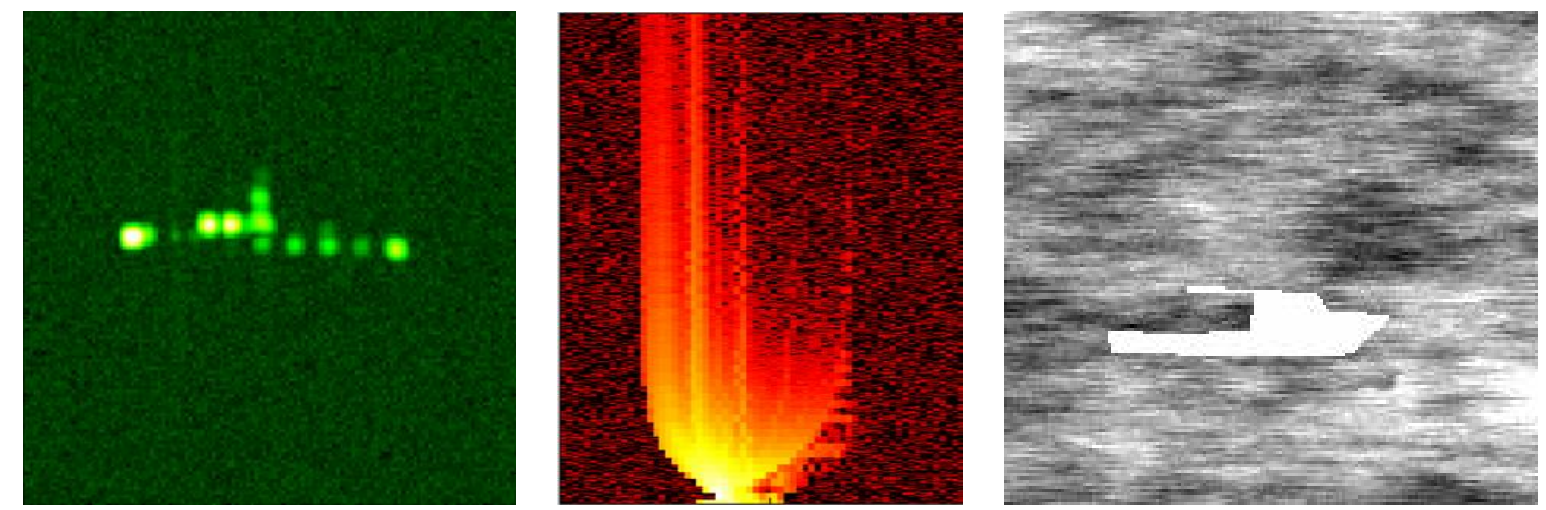

Figure 3.2. Simulated sensor data (Cabin boat) for the radar (ISAR image), left, (laser range profiles for the complete scenario, top is begin, end is bottom), middle, and infrared imagery (right).

For every 0.01 seconds in the scenario the position of the boats are determined. Radar RCS data are simulated for 64 frequency steps comprising in total $500 \mathrm{MHz}$. Applying a 2-dimensional inverse Fourier transform to 0.64 second of data, ISAR images of 64 by 64 pixels are obtained. A hamming window is also applied to reduce sidelobes. A speckle background (3 looks) corresponding to a sigma 0 of $-30 \mathrm{~dB}$, which is typical for sea state 3 (van den Broek et al, 2000), 
has been added to the image. The images have $30 \mathrm{~cm}$ range resolution, while cross-range resolution depends on the motion of the boat.

In the infrared images targets are seen from the front at large distances. Nearby, the targets are seen from the side, and are covered by more pixels. Therefore unlike radar and laser, more information is obtained at close range. In this scenario the boat is warmer compared to the sea, so that in principal most of the boat can be distinguished from the background. At larger distances this is more difficult. Since the facet model does not allow specification of thermal properties of small regions, the target apparent temperature is too constant, suggesting a better segmentation of the target than would be possible in reality. A correction is made for this for larger distances by adding uncertainties. Images are generated for every 0.64 seconds corresponding to the rate of the ISAR images.

Laser range profiles have been obtained for 72 range cells of $30 \mathrm{~cm}$ resolution for every 0.64 seconds. Noise has been added so that the signal at large distances $(>6000 \mathrm{~m}$ ) is dominated by noise. In Figure 3.2 (middle) the range profiles are plotted in the horizontal direction while the vertical axis indicates the time (or more or less the distance). The range profiles clearly show the bow (left) and the cabin (middle). Note that the range profiles become strong and short in the bottom of Figure 3.2, which corresponds to the end of the scenario. In this part of the scenario the boat is close to the coast and observed with azimuth angles near 90 degrees (i.e. line-of-sight perpendicular to the long axis of the boat) causing short and bright range profiles.

\subsection{Databases}

To classify the targets, the simulated data described in the previous section have to be compared with data in a database. Therefore also data for databases have been simulated using the models. To populate the database for the radar a standard scenario is considered with sea state 4, a mean elevation angle of 90 degrees, and for every 2 degrees of azimuth angle. For laser sensor the database consists of data for every 5 degrees of azimuth angle. For the infrared sensor database images are obtained at distances from 1 to $8 \mathrm{~km}$, with $6 \mathrm{~m}$ intervals and for every 15 degrees of azimuth angle from 0 to 90 degrees.

\subsection{Feature extraction and probabilities}

Comparison of the sensor data with data in the database is done using features extracted from the infrared and ISAR imagery and by correlation of the laser range profiles.

The extraction of features uses a detection mask which is obtained by applying a threshold. For the ISAR imagery this is done using a so-called CFAR detector (Novak and Hesse, 1991) which takes into account local variation in the backscattering of the background. In Figure 3.3 we show detection masks for the ISAR and infrared imagery which corresponds to the imagery shown in Figure 3.2.
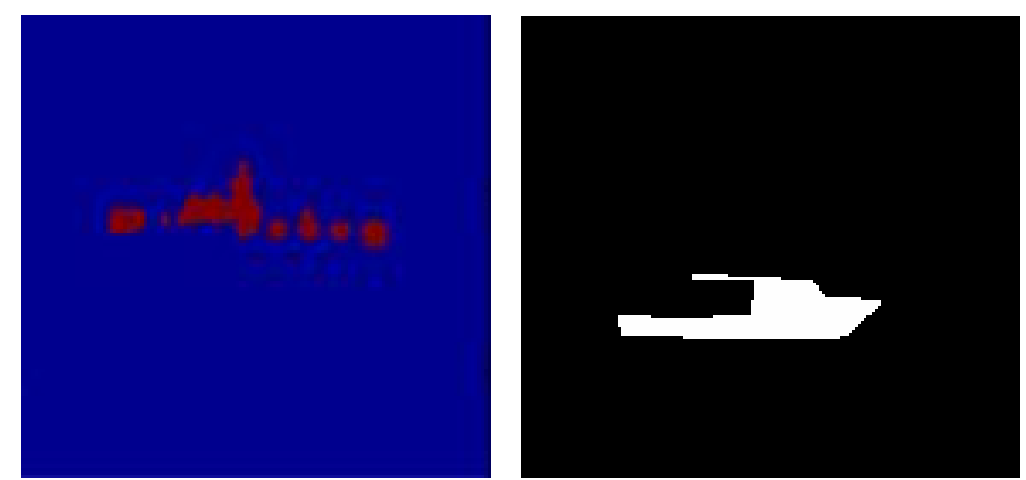

Figure 3.3. Detection masks for the ISAR (left) and infrared images (right) of the Cabin boat shown in Figure 3.2

Various features are calculated for the infrared and ISAR images using the pixels, which belong to the detection mask (van den Broek et al., 2003). These features are listed in table 3.2. For the infrared features, uncertainties are added to the features derived from the detection masks to allow more realistic variations. Uncertainties increase with distance, and also influence the detection probability at large distances. 
Table 3.2: Description of the features from the ISAR and infrared images

\begin{tabular}{|l|l|l|}
\hline $\begin{array}{l}\text { ISAR } \\
\text { image }\end{array}$ & Infrared image & Description \\
\hline Area & Area & Total area occupied by the pixels in the detection mask \\
\hline RCS & $\begin{array}{l}\text { Radar cross section: total backscatter power for the pixels in the } \\
\text { detection mask }\end{array}$ \\
\hline Length & Length & $\begin{array}{l}\text { Dimension of the long axis of the ship from the maximum } \\
\text { extension of the detection mask, corrected for the azimuth angle. }\end{array}$ \\
\hline & IntensityVariance & $\begin{array}{l}\text { Dimension of the long axis of the ship from the maximum } \\
\text { extension of the detection mask, estimated using distance from } \\
\text { radar }\end{array}$ \\
\hline & Height & $\begin{array}{l}\text { Maximum dimension of the vertical direction (height) of the } \\
\text { detection mask in the infrared image }\end{array}$ \\
\hline WFR & HeightVariance & Variance of the detection mask in vertical direction. \\
\hline CVAR & & $\begin{array}{l}\text { Weighted rank fill ratio: the ratio of the total power of the 20\% } \\
\text { brightest pixels and of all pixels in the detection mask (Kreithen et } \\
\text { al. 1993) }\end{array}$ \\
\hline
\end{tabular}

In Figure 3.4 we show the Area feature as function of distance for both the infrared and the ISAR images. The infrared Area feature is much smaller than that of the ISAR Area feature. This is because when the infrared camera sees a frontal view of the boat (determined by width and height), the radar 'sees' most of the extension of the boat (width and length). At closer range when the boat is seen from a different azimuth angle, the area for infrared increases, but for radar stays more or less the same. For the Rhib, only the hotter part where the motor is located is detected in the infrared.
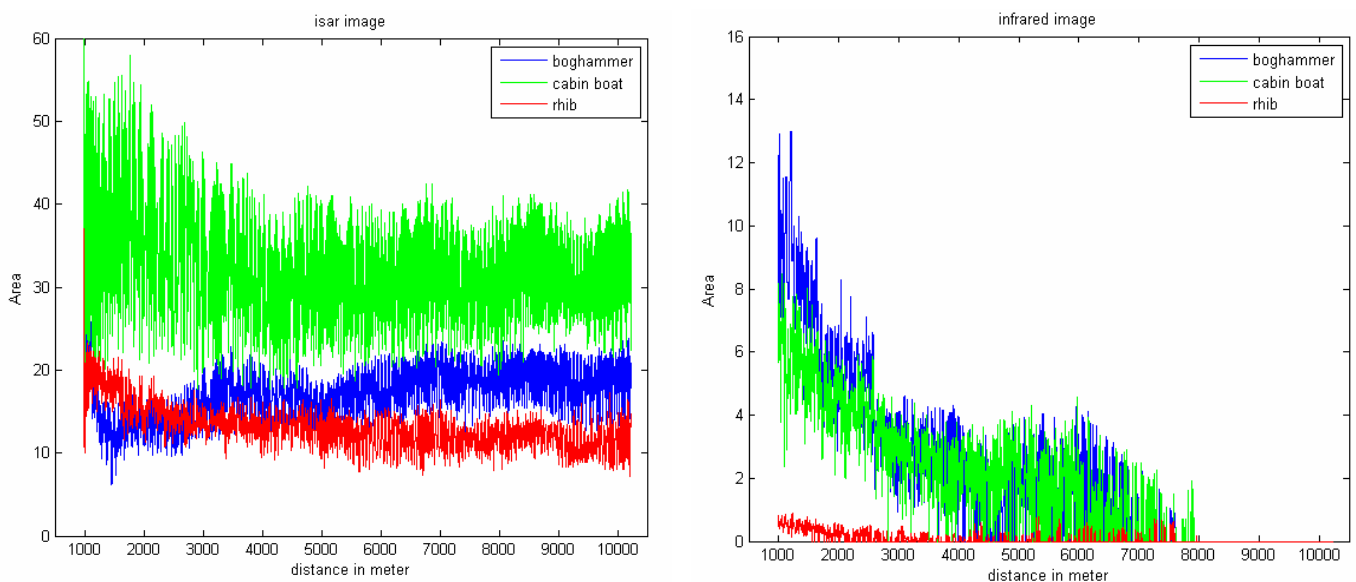

Figure 3.4. Area feature as function of distance for the ISAR (left, measuring top view) and infrared imagery (right, measuring observed 'side' view area).

To compare the features from the sensor images, comparable features are extracted from the images in the database, where the features are grouped for azimuth bins for the radar and azimuth-distance bins for the infrared. For comparison 
with the database appropriate azimuth bins and/or distance bins are selected, which correspond to the observation, using information which is assumed to be provided by the tracking radar. The various feature values can be considered as elements of multi-dimensional feature vectors in a multi-dimensional feature space. We show here an example of a twodimensional case (Figure 3.5), where the ISAR feature RCS is plotted against the ISAR feature Area for azimuth angles near 10 degrees. Also shown are ellipses which indicate the Mahalanobis distances (Duda and Hart, 1975), which are calculated from the covariance and the mean of the feature values and which characterize the distribution in feature space.

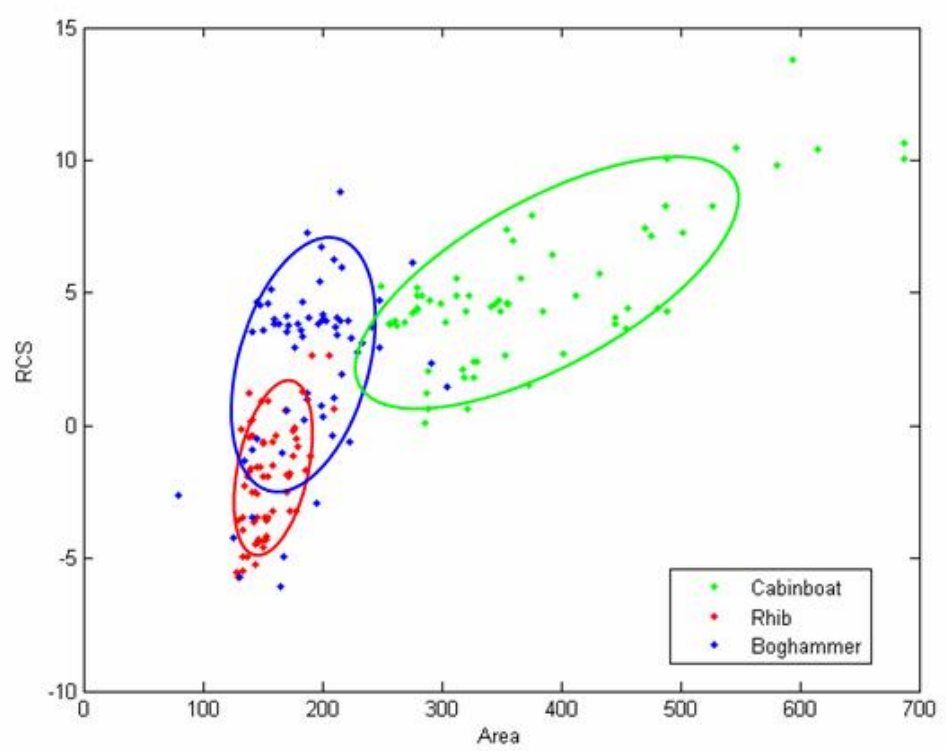

Figure 3.5. Two-dimensional feature space of RCS versus Area for azimuth angles of about 10 degrees. The ellipses represent points of equal Mahalanobis distance.

In fact we use 5 features for the radar and 5 features for the infrared sensor so that we actually deal with 5 dimensional feature spaces. For the three target classes we therefore construct 5-dimensional multi-variant target distributions. Using means and covariance matrices derived from these distributions and a feature vector $\vec{x}$ from the sensor image we calculate probabilities $P(\vec{x} \mid C)$ that this feature vector belongs to target class $\mathrm{C}$ (in this case Boghammer, Cabin boat or Rhib) following

$$
P(\vec{x} \mid C)=\frac{1}{2 \pi^{2 / 5} \Sigma_{C}^{-1}} \exp \left[-\frac{1}{2}\left(\vec{x}-\vec{\mu}_{C}\right)^{T} \Sigma_{C}^{-1}\left(\vec{x}-\vec{\mu}_{C}\right)\right]
$$

where $\vec{\mu}_{C}$ and $\Sigma_{C}$ are the mean and the covariance matrix for target class $C$ respectively.

We also calculate probabilities for target class 'other' comprising all other ships under consideration. This probability is given by

$$
P\left(\vec{x} \mid C_{u}\right)=\frac{1}{V}
$$

where $V$ is the volume of the feature space, which is believed to be representative for all other ships under consideration.

\subsection{Correlation}

For the laser we use correlation of laser range profiles to compare sensor data with data in the database. Since the aspect angle of the ship is known from the radar track data, the simulated "measured" laser range profile is correlated with the closest range profile (in aspect angle) in the database. The three correlations (for the three boats) give three likelihoods 
when taking the noise into account. A moderate correlation gives a high likelihood with large noise but a low one for little noise. These likelihoods are used to obtain probabilities using Bayes theorem. The target class 'other' is used here differently compared to the radar and the infrared camera. In this case the 'other' likelihood is obtained by calculating the confusion between the three boats and a constant estimate for the likelihood of a detected boat for the target class 'other'. We take here $30 \%$. Confusion is based on the product of likelihoods of two ships.

\section{SEA TARGET RECOGNITION}

In this section we present a method to classify ships on the basis of the probabilities described in the previous section. We introduce the classification method, present results for using single sensors and for the multi-sensor approach and finally describe a demonstrator on the basis of the method introduced in this paper.

\subsection{A priori probabilities and classification}

For the classification of the ships a Bayesian approach is followed. A priori information can be derived from expectation values based on historical records, but can also come from AIS information or by inspection of observed characteristics. For example when a ship of small dimensions has a relative slow variation in elevation angle with respect to the waves, this can be an indication for excessive load, which can be considered as anomalous behaviour. Also a ship with a high speed can pre-select certain ship classes, so that a priori chances have to be adapted. The probability $P(C \mid \vec{x})$ that we observe a certain target class $C$ given a feature vector $\vec{x}$ is in a Bayesian approach given by:

$$
P(C \mid \vec{x})=\frac{P(\vec{x} \mid C) P(C)}{P(\vec{x})}
$$

Where $P(C)$ are the a priori probabilities and $P(\vec{x})=\Sigma P(\vec{x} \mid C) P(C)$, with the summation $(\Sigma)$ over all target classes $C$. In practice, to determine a priori probabilities we first have to determine which class of ships are relevant. In the scenario we will consider as relevant class all boats with a small dimension $(<15$ meter). All ships larger than 15 meter will not be considered (see also Figure 4.1).

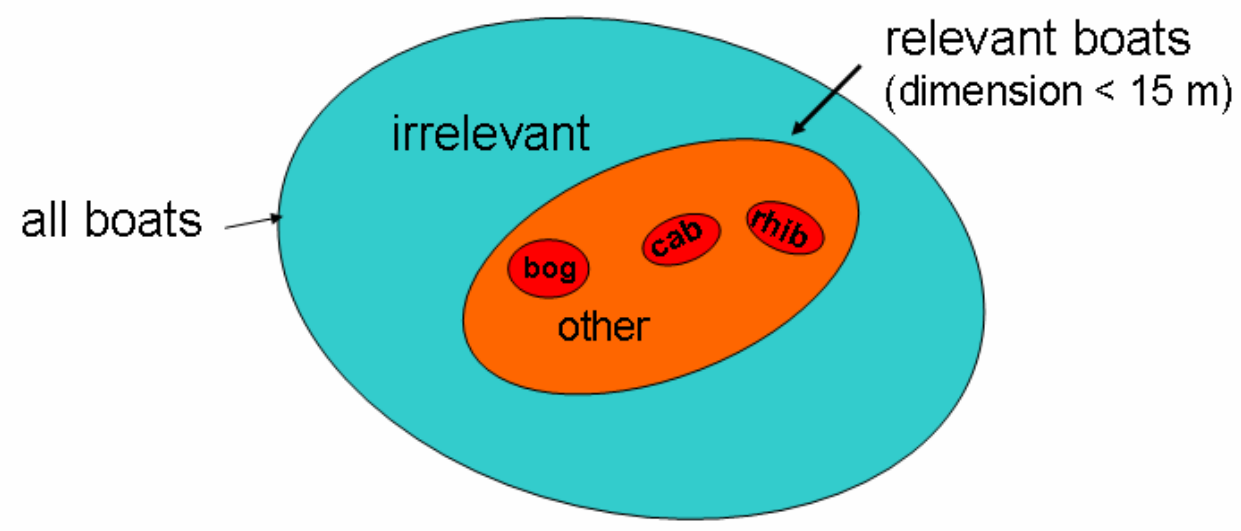

Figure 4.1. Schematic representation of the target classes

This implies that the volume of the feature space which has to be used to calculate the target class 'other' in Figure 4.1 in case of the radar and infrared is determined by the features belonging to this type of boat. For initial a priori probabilities we assume $15 \%$ of the detections of small boats is a Boghammer, Cabin boat or Rhib (all three equal chance) and $85 \%$ of detections is for other boats. Using abovementioned method and probabilities for equation 3 targets can now be classified given the observed images and profiles. 


\subsection{Updating of classification results}

After classification results have been obtained, based on a priori information, this information can be used to adjust the a priori probabilities for a subsequent classification. This is done by adding classification results in the next classification cycle. The a priori probabilities are adjusted with a 'coupling' factor to the classification results which is small $(<1 \%)$ so that fluctuations in the classification results due to low quality observations will not influence the final outcome too much. In Figure 4.2 this update cycle is visualized. Various values for the coupling factor can be used. For example a coupling factor of $0.1 \%$ implies that typically 10 minutes are needed before new classification results will become manifest, since in this scenario new results are obtained every 0.64 seconds.

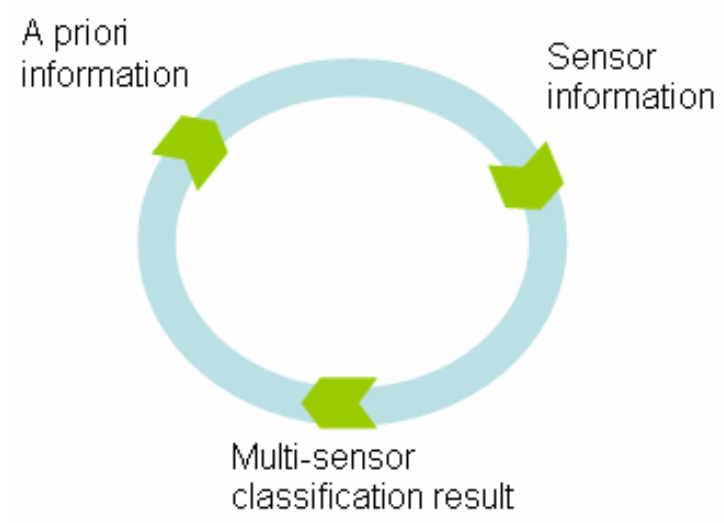

Figure 4.2. Schematic representation of the update cycle

\subsection{Single sensor classification}

Using the classification methods, the a priori probabilities, and the learning cycle described in the previous section we can calculate classification results per sensor as function of time (which in this scenario is equivalent to distance a function of distance). The coupling factor for the learning cycle is chosen to be $0.5 \%$. The results for the three sensors are shown in Figure 4.3.
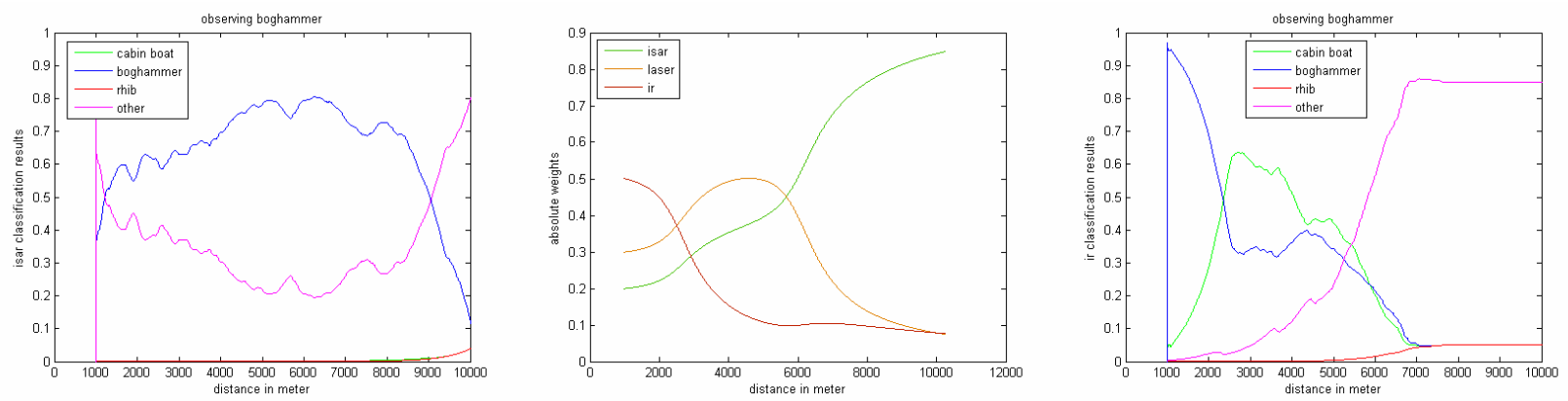

Figure 4.3. Single sensor classification results as function of distance observing the Boghammer: radar (left), laser (middle) and infrared camera (right).

Figure 4.3 shows that the radar can already indicate the Boghammer with a probability of $70 \%$ at $8.5 \mathrm{~km}$. When a boat approaches, the azimuth angle changes. At the end of the scenario the azimuth angle is about 90 degrees. In these cases the radar sees the width of the boats in range direction and has fewer possibilities for discrimination, so that the classification result for the Boghammer decreases. For the laser, correlation gives equal likelihood for each target class at the beginning of the scenario. However at distances $<8 \mathrm{~km}$ the likelihood for the Boghammer starts to dominate, so that the probability increases steadily and dominates for distances $<6 \mathrm{~km}$. For the infrared at distances over $8 \mathrm{~km}$ no detection takes place. For distances between $2.5 \mathrm{~km}$ and $8 \mathrm{~km}$ there is confusion between the Cabin boat and Boghammer, as in frontal view, they are alike. Only for distances smaller than $2.5 \mathrm{~km}$ the Boghammer is indicated with high probability, as the target is seen from aside and length is determined more accurately. The camera complements the other sensors, as it observes the target partly in a perpendicular direction. 


\subsection{Multi-sensor classification}

For the multi-sensor classification we have to determine the contributions of the single sensor classification. The quality of an observation from a sensor is however not constant, but is dependent on the distance, since the signal-to-noise ratio worsens with increasing distance for the radar and laser. Especially for distances larger than about $6 \mathrm{~km}$, noise is a dominant factor for the laser data. Noise is also a problem for the radar, but is only dominating for distances $>20 \mathrm{~km}$. For an observation tower of 40 meter height also horizon effects play a role for $>20 \mathrm{~km}$, so that this kind of surveillance is always limited to about $20-25 \mathrm{~km}$. For the infrared data the number of pixels on a target decreases with the square of the distance.

In order not to introduce too much uncertainty the contributions from the single sensors are therefore weighted with distance in the averaging to give a multi-sensor classification result. The contributions relative to the radar as function of distance are shown in the following figure (see Figure 4.4, left), where the contribution of the radar is considered to be constant for the distances $<10 \mathrm{~km}$. The relative contributions for the laser and infrared at short distance when laser and infrared are fully capable of contributing, are chosen to be 1.5 and 2.5 respectively.
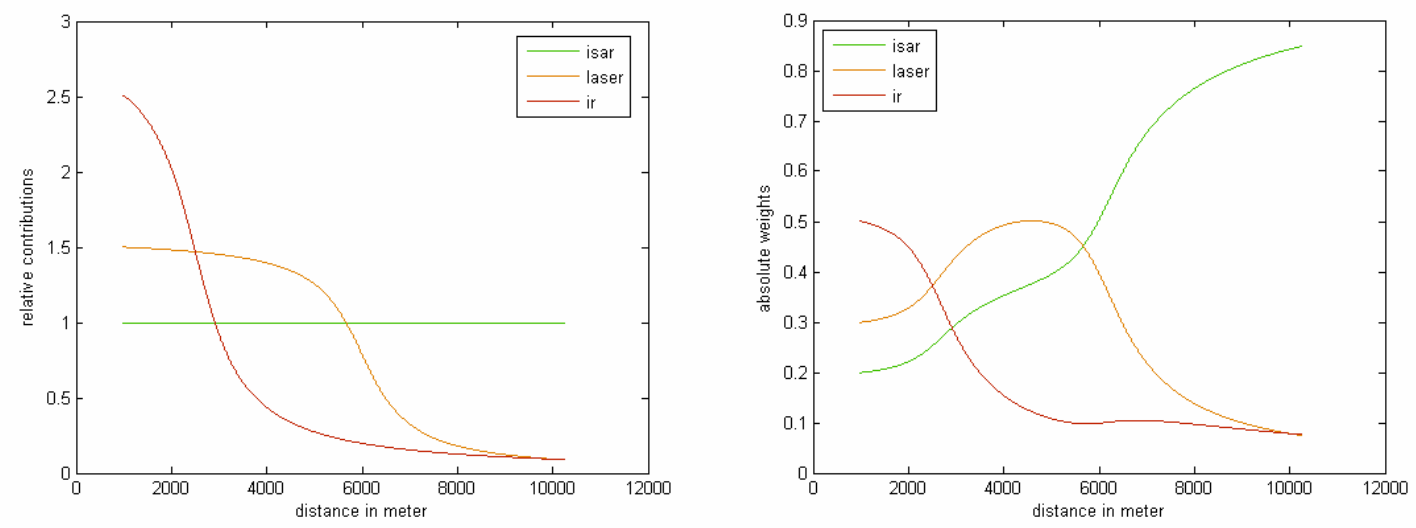

Figure 4.4 Weighting factors as a function of distance for the single sensors. To the left weighting factor relative to the radar contribution. To the right the absolute factors as used in the calculations

When we inspect the absolute weighting factors (see Figure 4.4, right), we see that the radar plays a dominant role for distances larger than $6 \mathrm{~km}$. The laser is dominating between 3-6 km and the infrared camera dominates at distances < 3 $\mathrm{km}$. Using the weighting factors we can determinate multi-sensor classification results for the scenario presented in section 2. In Figure 4.5 we present classification results as a function of distance when a Boghammer is observed. We present in Figure 4.5 results for a coupling factor of 0.1 and $0.5 \%$ (see Section 4.2).
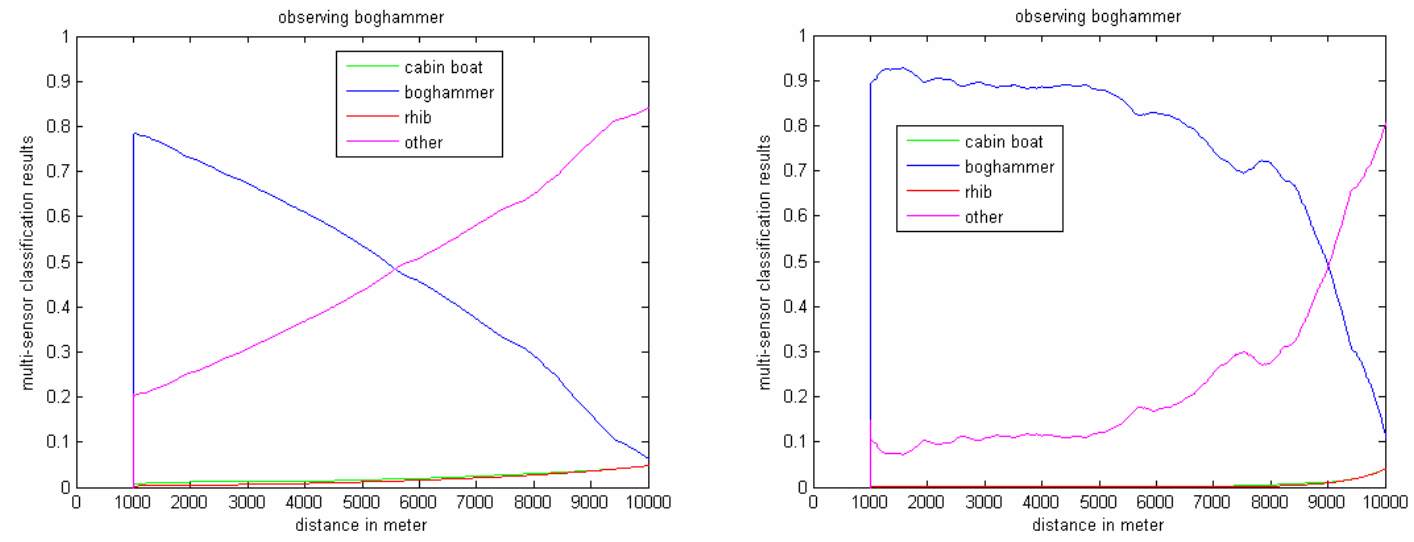

Figure 4.5. Multi-sensor classification results as a function of distance for a coupling factor of $0.1 \%$ (left) and $0.5 \%$ (right). 
The figure shows that the multi-sensor classification steadily increases for the Boghammer as it is approaching. The coupling factor should be not too small otherwise the multi-sensor results do not reflect the classification results which are for example obtained by the radar $(70 \%$ at $8000 \mathrm{~m})$. For a coupling factor of $0.5 \%$ we see multi-sensor classification is more directly coupled to the single sensor results. This also implies that decreasing radar classification results at the end of the scenario when the boats are observed with an azimuth angle of 90 degrees becomes noticeable in the multisensor classification results. Comparable results are obtained for the Rhib and Cabin boat.

\subsection{Demonstrator}

The process described in the previous section has been implemented in a demonstrator using MATLAB (http://www.mathworks.com/). Using this demonstrator the scenario can be simulated as a function of time, where it is also possible to change the time in the scenario interactively. Single sensor as well as multi-sensor classification results are presented. Also images and range profiles are shown, together with target information like location and several target features. The interface is shown in Figure 4.6

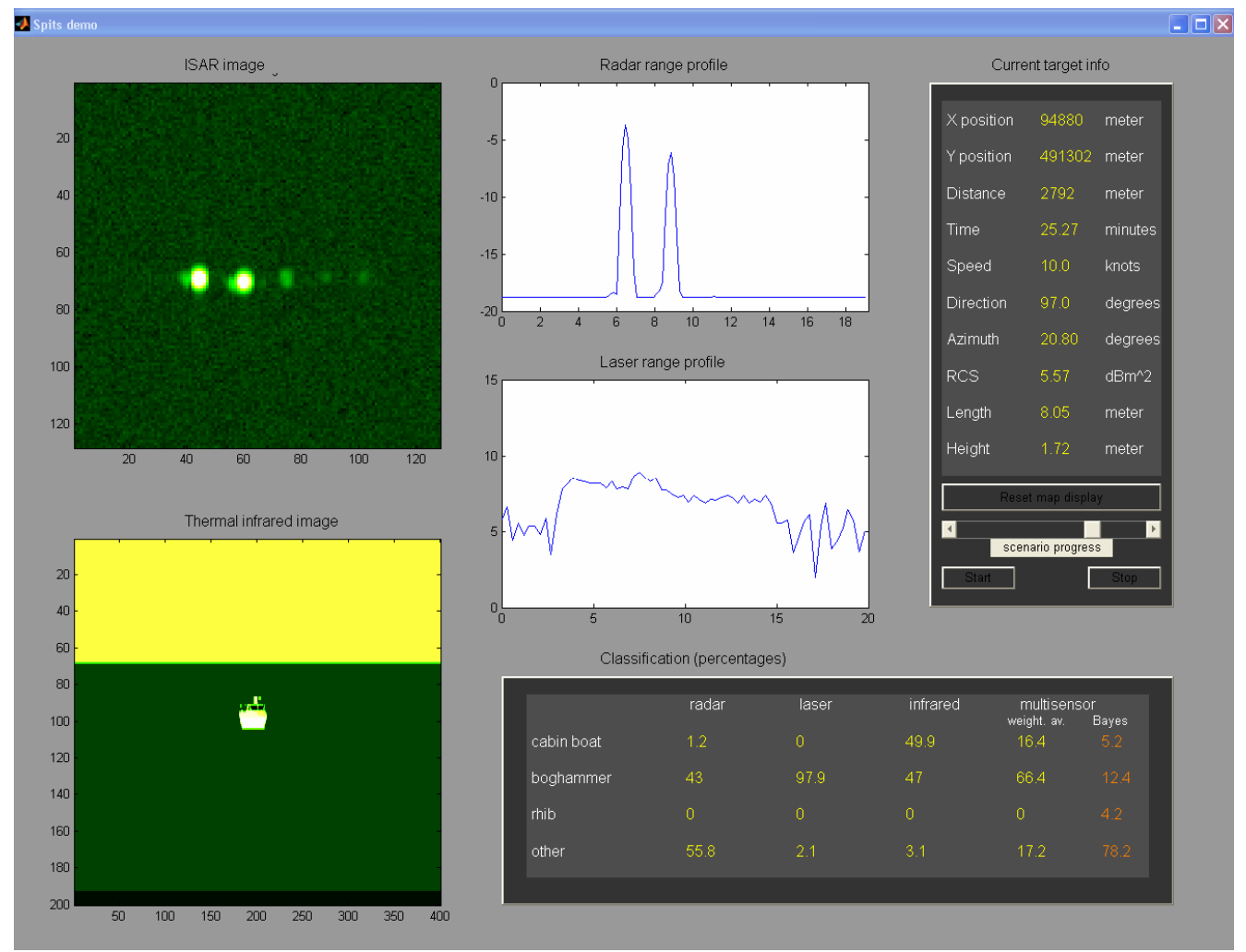

Figure 4.6. Interface of the multi-sensor classification demonstrator

\section{DISCUSSIONS AND CONCLUSIONS}

We have presented a concept for coastal surveillance. This concept has been evaluated and demonstrated using modelled sensor data. We give the following conclusions.

Different sensors give complementary information. Each sensor has its own specific distance range in which it contributes most to classification. The laser and radar give better opportunities for discrimination when the ship is observed along the long axis while the infrared gives more information when the ship is observed perpendicular to this.

The multi-sensor approach reduces the number of misclassifications. It is clear that the various sensors give different kind of information about a target. The radar and the laser reflection are dominated by geometrical structure of the target, while an infrared image reveals the apparent temperature distribution over the target. Combining different kinds of information can prevent that targets that are similar for one sensor will not be confused since the other sensor is probably able to discriminate between those targets. 
Reliable classification results are obtained at an earlier stage compared to a single sensor approach. For improving situational awareness in a coastal environment and to detect anomalous behaviour early knowledge about a target is a necessity. The radar is capable of providing information at large distances but is less able to provide definite conclusions about the ship's nature. The radar however can cue the laser and/or infrared sensor so that earlier more reliable target recognition is obtained.

For operational applications of the concepts and methods described in this paper also the atmospheric conditions have to be taken into account, since for example fog will reduce the distance at which the laser and infrared camera can contribute. Also at larger distances waves may shield the view on the target and reflection from waves can contribute to the radar return so that ISAR images become distorted.

Multi-sensor results are obtained by weighting the single sensor results. The weighting factors depend on the distance, since for larger distances the performance of the laser and infrared sensor decrease. When also atmospheric conditions will influence the performance it will be more appropriate to use quality factor for dynamically adapting weighting factors between the sensors. A comparable quality factor can be used for variation in azimuth angle to account for the complementary information produced by the radar and laser compared to the infrared camera for different azimuth angles.

Another factor of importance is the database. For good classification results a detailed database is needed for all ships which are of interest. For the radar and laser it is needed to divide the database by azimuth angle, while for the infrared camera the database should be divided by both azimuth and distance. In practice it will be difficult to obtain such a database. One option may be that monitoring results are used for the database after validating the classification. Another possibility is to use modelling results, which however first have to be checked by real measurements.

\section{REFERENCES}

1. Brand, M.G.E., 1995, Radar signature Analysis and Prediction by Physical Optics and Ray Tracing, the RAPPORT code for RCS prediction, TNO report FEL-95-A097.

2. Broek, van den, A.C., Dekker, R.J., Steeghs, T.P.H., 2003, Robustness of Features for Automatic Target Discriminaton in High Resolution Polarimetric SAR Data, Algorithms for Synthetic Aperture Radar Imagery X, Proc. of SPIE, Vol. 5095, pp. 242-253.

3. Duda, R.O., Hart, P.E., 1973, Pattern Classification and Scene Analysis, John Wiley and Sons, New York.

4. Ewijk, van, L.J., 1992, Technical formulation of the high frequency Radar Cross Section prediction model, TNO report FEL-92-A286.

5. Gordon, W.B., 1975, Far field approximation to the Kirchoff-Hclmholtz representations of scattered fields, IEEE Transactions on Antennas and Propagation, Vol.23, pp.590-592

6. Knott, E.F. , 1981, A tool for predicting the Radar Cross Section of an arbitrary trihedral corner, IEEE South East Conference, pp. 17-20

7. Kunz, G.K., M.A.C. Degache, M.M. Moerman, A.M.J. van Eijk, F.P. Neele, S.M. Doss-Hammel and D. Tsintikidis, 2004, Status and developments in EOSTAR, a model to predict IR sensor performance in the marine environment, SPIE's 11th International Symposium on Remote Sensing,Optics in Atmospheric Propagation and Adaptive Systems VII, Maspalomas, Gran Canaria, September 2004.

8. Levanon, N., 1988, Radar principles, John Wiley and Sons, New York.

9. Neele, F.P., 2005, Infrared ship signature prediction, model validation and sky radiance, SPIE Proc. Vol. 5811-24.

10. Novak, L.M., S.R. Hesse, S.R., 1991, On the performance of order-statistics CFAR detectors, IEEE Conference Record of the $25^{\text {th }}$ Asilomar Conference on Signals, Systems and Computers, Vol.2, pp. 835-840.

11. Schwering, P.B.W., 2006, IRST evaluation methodologies: Maritime infrared background simulator, SPIE Proc. Vol. 6206, 620621, 2006

12. Theil, A., Huizing, A.G., van Heijningen, A.W.P., 2006, Single Picture Integration for Territorial Waters Surveillance (SPITS), An initiative to improve situational awareness in littoral waters, MAST (Maritime Systems and Technology) Conference, Nice France, 4-6 September 2006

13. van den Broek, S.P., Bakker, E.J., de Lange, D.J., Theil, A, 2000, Detection and classification of infrared decoys and small targets in a sea background, SPIE Vol. 4029, 70-80. 